14,15

\title{
Лазерная нанофлюидика жидких кристаллов
}

\author{
(C) Izabela Śliwa ${ }^{1}$, A.B. Захаров ${ }^{2,9}$ \\ ${ }^{1}$ Poznan University of Economics and Business, \\ Poznan, Poland \\ ${ }^{2}$ Институт проблем машиноведения РАН, \\ Санкт-Петербург, Россия \\ ฯ E-mail: izabela.sliwa@ue.poznan.pl, alexandre.zakharov@yahoo.ca
}

Поступила в Редакцию 13 января 2020 г.

В окончательной редакции 13 января 2019 г.

Принята к публикации 14 января 2019 г.

\begin{abstract}
Численными методами, в рамках нелинейного обобщения классической теории Эриксена-Лесли, допускающей учет термомеханических вкладов как в выражение для сдвигового напряжения, так и в уравнение баланса энтропии, описано несколько сценариев формирования гидродинамических течений в наноразмерных планарно-ориентированных жидкокристаллических (ПОЖК)-каналах. В результате формирования как градиента температуры $\nabla T$, в изначально однородно прогретом ПОЖК-канале под действием сфокусированного лазерного излучения, так и градиента поля директора $\nabla \hat{\mathbf{n}}$, под действием статического электрического поля, естественно возникающего на границе раздела ЖК-фаза/твердое тело, и в результате взаимодействия $\nabla T$ и $\nabla \hat{\mathbf{n}}$, в наноразмерном ПОЖК-канале может окончательно сформироваться вихревой поток.
\end{abstract}

Ключевые слова: физика жидких кристаллов, нанофлюидика, термомеханические силы.

DOI: 10.21883/FTT.2020.06.49359.002

\section{1. Введение}

Интерес со стороны исследователей к нанофлюидике, то есть, области физики жидкостей в нанолитровых объемах $\left(1 \mathrm{nl}=10^{-12} \mathrm{~m}^{3}\right)$, продиктован многообещающими приложениями этих систем в биологии [1], оптоэлектронике [2] и различных сенсорах и датчиках [3], в основу которых положены анизотропные молекулярные жидкости и жидкокристаллические (ЖК) материалы. Часто манипуляции такими наноразмерными анизотропными молекулярными системами в сверхтонких капиллярах и каналах осуществляются с помощью внешнего электрического поля [4]. Этот метод транспортировки нанолитровых объемов одинаково применим как для молекулярных жидкостей, так и для манипулирования нанолитровыми объемами ЖК-материалов. Отличительной особенностью ЖК-систем от анизотропных молекулярных жидкостей является то, что при определенных термодинамических условиях в ЖК-системах формируется ориентационное упорядочение молекул, которое

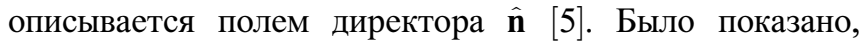
что взаимодействие градиентов поля директора $\nabla \hat{\mathbf{n}}$ и температуры $\nabla T$ ответственно за возникновение термомеханической силы (ТМС), которая, в свою очередь, ответственна за формирование устойчивого гидродинамического потока $\mathbf{v}$ ЖК-материалов в тонких и сверхтонких каналах и капиллярах [6]. Величина этого потока пропорциональна сдвиговой составляющей термомеханического вклада $\sigma_{z x}^{t m}$ в тензор напряжения (ТН) $v \sim \frac{d}{\eta} \sigma_{z x}^{t m}$, где $\eta-$ сдвиговая вязкость ЖК-материала, а $d$ - ширина ЖК-канала. Таким образом, необходимым и достаточным условием возникновения направленного потока ЖК-материала в сверхтонких каналах и капиллярах под действием ТМС является наличие градиентов поля директора $\nabla \hat{\mathbf{n}}$ и температуры $\nabla T$. Если локальный градиент температуры в объеме ЖК-фазы легко формируется с помощью лазерного излучения, то градиент поля директора удается сформировать, например, посредством гибридной ориентации ЖК-фазы, в которой ориентация поля директора на одной из поверхностей планарная, а на другой - гомеотропная. Все это указывает на то, что существует возможность немеханической транспортировки нанолитровых объемов ЖК-материала под действием сфокусированного лазерного излучения. В случае однородно-ориентированной ЖК-фазы, когда ориентация поля директора на ограничивающих поверхностях либо планарная, либо гомеотропная, направленное течение ЖК-фазы под действием градиента температуры отсутствует. В этом случае чтобы инициировать горизонтальное течение ЖК-фазы, необходимо деформировать однородно ориентированную ЖК-среду, с тем чтобы создать градиент поля директора. Этим фактором, который позволит деформировать планарноориентированную ЖК-полость, заключенную между двумя ограничивающими поверхностями, может служить перпендикулярно направленное электрическое поле. Таким полем может являться электростатическое поле двойного электрического слоя, естественно возникающего на границе раздела ЖК-фаза/твердое тело. Поскольку ЖК представляет собой слабый электролит, в котором число ионов практически равно числу катионов, то в пристенном слое на границе раздела ЖК-фаза/твердое 
тело возникает двойной электрический слой с плотностью заряда $\sigma$. Электрическое поле, создаваемое этим зарядом, пронизывает ЖК-фазу на расстояние порядка длины дебаевской экранировки $\lambda_{D}[7]$ и способно деформировать пристенные слои однородно-ориентированной ЖК-полости. При этом возникает вопрос: какой должна быть плотность заряда двойного электрического слоя, для того чтобы осуществить деформацию ЖК-фазы и тем самым инициировать горизонтальный поток ЖК-материала в тонком капилляре или канале.

Исследование этих новых состояний будет проведено в рамках нелинейного обобщения классической теории Эриксена-Лесли $[8,9]$ с учетом балансов массы, импульсов и моментов, действующих на единицу объема ЖК-материала, а также с учетом баланса энтропии [10]. Численными методами будут исследованы различные гидродинамические режимы формирования течений в наноразмерных планарно-ориентированных ЖК-полостях, заключенных между двумя параллельными границами, под действием градиента температуры и электростатического поля, естественно возникающего на границе раздела ЖК-фаза/твердое тело.

\section{2. Основные уравнения}

Рассмотрим длинный прямоугольный ЖК-канал с размерами $L$ и $d(L \gg d)$, ограниченный твердыми горизонтальными и вертикальными поверхностями. Допустим, что директор планарно ориентирован на всех поверхностях. Рассмотрим систему координат, отсчитываемую от нижней ограничивающей поверхности ЖК-канала так, что ось $X$ и орт $\hat{\mathbf{i}}$ совпадают с направлением директора на нижней (верхней) ограничивающей поверхности $\left(\hat{\mathbf{i}} \| \hat{\mathbf{n}}_{\mathrm{z}=-\mathrm{d}}\right)$, в то время как ось $Z$ и орт $\hat{\mathbf{k}}$ направлены ортогонально $\left(\hat{\mathbf{k}} \perp \hat{\mathbf{n}}_{\mathrm{z}=-\mathrm{d}}\right)$, а орт $\hat{\mathbf{j}}=\hat{\mathbf{k}} \times \hat{\mathbf{i}}$. Будем предполагать, что переориентация поля директора $\hat{\mathbf{n}}=\left(n_{\mathrm{x}}, 0, n_{\mathrm{z}}\right)=\sin \theta \hat{\mathbf{i}}+\cos \theta \hat{\mathbf{k}}$ под действием градиента температуры $\nabla T$, формирующегося за счет сфокусированного лазерного излучения и плотности заряда двойного электрического слоя $\sigma$, осуществляется в плоскости $\mathrm{XZ}$. Здесь $\theta \equiv \theta(x, z, t)$ - полярный угол, образованный директором $\hat{\mathbf{n}}$ и ортом $\hat{\mathbf{k}}$.

В случае когда нематик находится в контакте с твердой ограничивающей поверхностью, то имеет место избирательная адсорбция ионов. Так например, положительные ионы притягиваются поверхностью, в то время как отрицательные ионы (катионы) отталкиваются. Принимая во внимание тот факт, что ЖК-фаза обычно содержит практически равное количество ионов и катионов (слабый электролит), то находясь в контакте с твердой ограничивающей поверхностью образуется двойной электрический слой с плотностью заряда $\sigma$. Электростатическое поле $\mathbf{E}$, инициируемое плотностью электрического заряда $\sigma$, проникает в объем ЖК-фазы на глубину порядка дебаевской длины экранировки
$\lambda_{\mathrm{D}}=\sqrt{\frac{\epsilon \epsilon_{0} k_{B} T}{2 e^{2} n_{e q}}}$ и может быть рассчитано в рамках теории Пуассона-Больцмана как [7]

$$
\mathbf{E}(x, z)=E_{x} \hat{\mathbf{i}}+E_{z} \hat{\mathbf{k}},
$$

где компоненты электрического поля имеют вид

$$
E_{x}=E_{0}\left[\exp \left(-\frac{x}{\lambda_{\mathrm{D}}}\right)-\exp \left(-\frac{L-x}{\lambda_{\mathrm{D}}}\right)\right]
$$

и

$$
E_{z}=E_{0}\left[\exp \left(-\frac{z}{\lambda_{\mathrm{D}}}\right)-\exp \left(-\frac{d-z}{\lambda_{\mathrm{D}}}\right)\right] .
$$

соответственно, а $E_{0}=\sigma /\left(\epsilon_{0} \epsilon_{a}\right)$ - поверхностное электростатическое поле, $\epsilon_{0}-$ диэлектрическая проницаемость вакуума, $\epsilon_{a}=\left(\epsilon_{\|}+2 \epsilon_{\perp}\right) / 3$ - диэлектрическая проницаемость ЖК-среды, $\epsilon_{\|}$и $\epsilon_{\perp}-$ диэлектрические постоянные вдоль и поперек направления директора $\hat{\mathbf{n}}$ соответственно, $\epsilon=\epsilon_{\|} \cos ^{2} \theta_{s}+\epsilon_{\perp} \sin ^{2} \theta_{s}, e-$ заряд протона, $k_{B}$ - постоянная Больцмана, $\theta_{s}-$ значение угла между направлением директора $\hat{\mathbf{n}}_{s}$ на ограничивающей поверхности и ортом $\hat{\mathbf{k}}$ и $n_{e q}-$ концентрация ионов в объеме ЖК-фазы.

Наша главная цель состоит в том, чтобы исследовать влияние локально формирующегося, с помощью лазерного излучения, градиента температуры $\nabla T$ в объеме ЖК-фазы и градиента поля директора $\nabla \hat{\mathbf{n}}$, формирующегося под влиянием электростатического поля $\mathbf{E}$, инициируемого плотностью электрического заряда $\sigma$, на характер гидродинамического потока в наноразмерном планарно-ориентированном ЖК (ПОЖК)канале. В нашем случае верхняя ограничивающая поверхность нагревается инфракрасным лазерным лучом, причем длительность и мощность лазерного импульса ограничена только тем условием, чтобы температура верхней ограничительной поверхности находилась в диапазоне температур, соответствующих существованию нематической фазы. В настоящей работе предлагается новый подход, описывающий механизм формирования сложного гидродинамического течения в наноразмерных ПОЖК-каналах под действием потока тепла

$$
\lambda_{\perp}\left(\frac{\partial T(x, z, t)}{\partial z}\right)_{0 \leq x \leq L, z=d}=\mathrm{q},
$$

направленного ортогонально $\mathbf{q}=q \hat{\mathbf{k}}$ через верхнюю ограничивающую поверхность канала в ЖК-фазу, в то время как на остальных поверхностях ЖК-канала поддерживается постоянная температура

$$
T_{0 \leq x \leq L, z=0}=T_{x=0,0<z<d}=T_{x=L, 0<z<d}=T_{w} .
$$

Здесь $\lambda_{\perp}$ - коэффициенты теплопроводности ЖК-фазы в направлении, перпендикулярном направлению директоpa $\hat{\mathbf{n}}$.

Следует отметить, что планарная ориентация ЖК-канала предполагает, что на нижней, верхней и двух 
боковых ограничивающих поверхностях выполнены следующие граничные условия для поля директора

$$
\begin{aligned}
& \left(n_{x}\right)_{0 \leq x<L, z=0}=\left(n_{x}\right)_{0 \leq x<L, z=d}=1, \\
& \left(n_{x}\right)_{x=0,0 \leq z<d}=\left(n_{x}\right)_{x=L, 0 \leq z<d}=1 .
\end{aligned}
$$

В свою очередь, поле скорости $\mathbf{v}=u \hat{\mathbf{i}}+w \hat{\mathbf{k}}$ удовлетворяет условию прилипания

$$
\begin{aligned}
& u_{0 \leq x<L, z=0}=u_{0 \leq x<L, z=d}=u_{x=0,0 \leq z<d}=u_{x=L, 0 \leq z<d}=0, \\
& w_{0 \leq x<L, z=0}=w_{0 \leq x<L, z=d}=w_{x=0,0 \leq z<d}=w_{x=L, 0 \leq z<d}=0,
\end{aligned}
$$

где $u \equiv v_{x}(x, z, t)$ и $w \equiv v_{z}(x, z, t)$ - компоненты вектора $\mathbf{v}$.

Принимая во внимание тот факт, что ширина ЖК-канала варьируется в пределах нескольких сотен нанометров, будем считать, что плотность ЖК-фазы постоянна по сечению канала $(\rho=$ const). Таким образом, мы имеем дело с несжимаемой ЖК-фазой, и условие несжимаемости $\nabla \cdot \mathbf{v}=0$ принимает вид

$$
u_{, x}+w_{, z}=0
$$

где $u_{, x}=\frac{\partial u}{\partial x}$.

В нашем случае гидродинамические уравнения, описывающие эволюцию как поля директора $\hat{\mathbf{n}}$ и скорости $\mathbf{v}$, так и поля температуры $T$ могут быть получены из условия баланса угловых и линейных моментов, действующих на единицу объема ЖК-фазы $[8,9]$, а также баланса энтропии [10]. Так, уравнение баланса угловых моментов имеет вид [7]

$$
\mathbf{T}_{\mathrm{el}}+\mathbf{T}_{\text {elast }}+\mathbf{T}_{\mathrm{vis}}+\mathbf{T}_{\mathrm{tm}}=0,
$$

где $\mathbf{T}_{\mathrm{el}}=-\epsilon_{0} \epsilon_{a} \mathbf{E} \times \hat{\mathbf{n}}(\mathbf{E} \cdot \hat{\mathbf{n}})-$ электрический, $\mathbf{T}_{\text {elast }}=$ $=\frac{\delta \mathscr{W}_{\mathrm{F}}}{\delta \hat{\mathbf{n}}} \times \hat{\mathbf{n}}-$ упругий, $\mathbf{T}_{\text {vis }}=\frac{\delta \mathscr{R}^{\text {vis }}}{\delta \hat{\mathbf{n}}_{, t}} \times \hat{\mathbf{n}}-$ вязкий и $\mathbf{T}_{\mathrm{tm}}=\frac{\delta \mathscr{R}^{\mathrm{tm}}}{\delta \hat{\mathbf{n}}_{\mathrm{t}}} \times \hat{\mathbf{n}}-$ термомеханический вклады в баланс угловых моментов соответственно (детали вычислений даны в Приложении), в то время как уравнение НавьеСтокса для поля скорости $\mathbf{v}$ принимает вид [7]

$$
\rho \frac{d \mathbf{v}}{d t}=\nabla \cdot \sigma
$$

где $\sigma=\sigma^{\text {elast }}+\sigma^{\text {vis }}+\sigma^{\text {tm }}-\mathscr{P Y}-$ полный тензор напряжения (ТН), в то время как его упругий, вязкий и термомеханический вклады имеют вид $\sigma^{\mathrm{el}}=-\frac{\partial \mathscr{W}_{F}}{\partial \nabla \hat{\mathbf{n}}} \cdot(\nabla \hat{\mathbf{n}})^{\mathrm{T}}, \sigma^{\text {vis }}=\frac{\delta \mathscr{R}^{\mathrm{vis}}}{\delta \nabla \mathbf{v}}$ и $\sigma^{\mathrm{tm}}=\frac{\delta \mathscr{R}^{\mathrm{tm}}}{\delta \nabla \mathbf{v}}$ соответственно, а $\hat{\mathbf{n}}_{, t}=\frac{d \hat{\mathbf{n}}}{d t}-$ материальная производная поля директора $\hat{\mathbf{n}}$. Здесь $\mathscr{R}=\mathscr{R}^{\mathrm{vis}}+\mathscr{R}^{\mathrm{tm}}+\mathscr{R}^{\mathrm{th}}-$ полная диссипационная функция Рэлея, в то время как $\mathscr{W}_{\mathrm{F}}=\frac{1}{2}\left[K_{1}(\nabla \cdot \hat{\mathbf{n}})^{2}+K_{3}(\hat{\mathbf{n}} \times \nabla \times \hat{\mathbf{n}})^{2}\right]-$ представляет собой плотность упругой энергии, а $K_{1}$ и $K_{3}-$ коэффициенты упругости продольного и поперечного изгибов соответственно. В свою очередь $\mathscr{P}$ представляет собой гидростатическое давление в планарноориентированном ЖК-канале.

В случае прогревания верхней ограничивающей поверхности инфракрасным лазерным лучом, изменения поля температуры $T(x, z, t)$ удовлетворяют уравнению теплопроводности [10]

$$
\rho C_{P} \frac{d T}{d t}=-\nabla \cdot \mathbf{q},
$$

где $\mathbf{q}=-T \frac{\delta \mathscr{R}}{\delta \nabla T}-$ тепловой поток через верхнюю ограничивающую поверхность в ЖК-фазу, а $C_{P}-$ коэффициент теплоемкости нематика.

Для того чтобы исследовать эволюцию поля директора $\hat{\mathbf{n}}$ (или полярного угла $\theta(x, z, t))$ к его равновесному распределению по сечению ПОЖК-канала $\hat{\mathbf{n}}_{\mathrm{eq}}(x, z)$, а также поля скорости $\mathbf{v}(x, z, t)$, вызванную взаимодействием градиентов поля директора $\nabla \hat{\mathbf{n}}$ и температуры $\nabla T$, мы рассмотрим безразмерные аналоги уравнений (7)-(9). Так, безразмерное уравнение баланса моментов может быть записано в виде (детали изложены в Приложении)

$$
\begin{aligned}
& \theta_{, \tau}=-\frac{L}{d}\left(1+\gamma_{12} \cos 2 \theta\right) u_{, z}-\frac{E_{z}^{2}}{2} \sin 2 \theta+\delta_{1}\left[g(\theta) \theta_{, z z}\right. \\
& \left.+\frac{1}{2} \sin 2 \theta \mathscr{K}\left(\theta_{, z}^{2}-\frac{2 d}{L} \theta_{, x z}\right)-\frac{2 d}{L} \theta_{, x} \theta_{, z} \mathscr{K} \cos 2 \theta\right] \\
& -\delta_{2}\left[\frac{d}{4 L} \chi_{, x} \theta_{, z} \sin 2 \theta+\frac{d}{2 L} \chi_{, z} \theta_{, x} \sin 2 \theta\left(\frac{1}{2}+\cos ^{2} \theta\right)\right] \\
& +\delta_{2}\left[\chi_{, z} 0_{, z}\left(1+\frac{1}{2} \sin ^{2} \theta\right)\right]
\end{aligned}
$$

где $\bar{x}=x / L$ и $\bar{z}=z / d-$ безразмерные пространственные переменные, $\tau=\frac{\epsilon_{0} \epsilon_{a} E_{0}}{\gamma_{1}} t-$ безразмерное время, $\mathscr{K}=1-K_{31}, g(\theta)=\sin ^{2} \theta+K_{31} \cos ^{2} \theta-$ функция полярного угла $\theta, K_{31}=K_{3} / K_{1}, \gamma_{21}=\gamma_{2} / \gamma_{1}, \delta_{1}=\left(\frac{E_{\mathrm{th}}}{\pi E_{0}}\right)^{2}$ и $\delta_{2}=\xi \frac{T_{N I}}{K_{1}} \delta_{1}-$ четыре параметра ЖК-системы, $E_{\mathrm{th}}=\frac{\pi}{d} \sqrt{\frac{K_{1}}{\epsilon_{0} \epsilon_{a}}}-$ пороговое напряжение электрического поля $\mathbf{E}, \xi \sim 10^{-12} \mathrm{~J} / \mathrm{mK}-$ термомеханическая постоянная [6], $\gamma_{1}$ и $\gamma_{2}-$ коэффициенты вращательной вязкости, а $T_{N I}$ - температура фазового перехода нематикизотропная фаза. Следует отметить, что в дальнейшем верхняя черта в обозначениях пространственных переменных $x$ и $z$ будет опущена.

Безразмерный аналог уравнения Навье-Стокса в нашем случае может быть записан в виде (детали изложены в Приложении)

$$
\begin{aligned}
& \delta_{3} \frac{d u}{d \tau}=\frac{d}{L}\left[\left(u_{, z} \mathscr{F}_{1}\right)_{, x}+\delta_{2} \chi_{, z} \theta_{, z} \mathscr{F}_{2}\right]_{, z} \\
& +\left[u_{, z} \mathscr{F}_{3}+\frac{d}{L}\left(\frac{\alpha_{1}}{\gamma_{1}} \sin 4 \theta w_{, z}-\frac{1}{2}\left(1-\gamma_{21} \cos 2 \theta\right) \dot{\theta}\right)\right]_{, z},
\end{aligned}
$$




$$
\begin{aligned}
\delta_{3} \frac{d w}{d \tau}= & \left(-w_{, z} \mathscr{F}_{6}-\frac{1}{2} \gamma_{21} \sin 2 \theta \dot{\theta}+\frac{L}{d} u_{, z} \mathscr{F}_{5}\right)_{, z} \\
& +\left(u_{, z} \mathscr{F}_{4}\right)_{, x}+(\mathscr{P}+\mathscr{B})_{, z},
\end{aligned}
$$

где множество функций $\mathscr{F}_{i}(i=1, \ldots, 6)$ и $\mathscr{B}$ имеют вид:

$$
\begin{gathered}
\mathscr{F}_{1}=\frac{1}{2 \gamma_{1}} \sin 2 \theta\left(\alpha_{1} \sin ^{2} \theta+\frac{\alpha_{5}+\alpha_{6}}{4}-\frac{\gamma_{2}}{2}\right), \\
\mathscr{F}_{2}=\frac{1}{4}\left(\sin 2 \theta-6 \sin ^{2} \theta-3 \cos ^{4} \theta-\frac{1}{2} \sin ^{2} 2 \theta\right), \\
\mathscr{F}_{3}=\frac{1}{4 \gamma_{1}}\left[\gamma_{1}+\gamma_{2} \cos 2 \theta+2 \alpha_{4}+2\left(\alpha_{5}+\alpha_{6}\right) \sin 2 \theta\right], \\
\mathscr{F}_{4}=\mathscr{F}_{1}+\frac{1}{2} \gamma_{21} \cos 2 \theta-\frac{1}{2}, \\
\mathscr{F}_{6}=-\mathscr{F}_{1}+\frac{\sin 2 \theta}{4 \gamma_{1}}\left(\alpha_{5}+\alpha_{6}+2 \alpha_{1} \sin 2 \theta\right), \\
\gamma_{1}\left(2 \alpha_{1}+\alpha_{5}+\alpha_{6}-\alpha_{1} \cos 2 \theta\right),
\end{gathered}
$$

и

$$
\mathscr{B}=\left[\frac{1}{2} \delta_{2} \chi_{, z} \theta_{, z} \sin 2 \theta\left(1+\frac{1}{4} \sin ^{2} \theta\right)-\delta_{1} g(\theta) \theta_{, z}^{2}\right]_{, z}
$$

соответственно, $\dot{\theta}=\frac{d \theta}{d \tau}-$ материальная производная полярного угла, а $\delta_{3}=\rho \frac{K_{1}}{\gamma_{1}^{2}} \delta_{1}^{2}-$ еще один параметр ЖК-системы. Следует отметить, что уравнение (12) было переписано с учетом условия несжимаемости $u_{, x}=-w_{, z}$. В свою очередь, безразмерное уравнение баланса энтропии принимает вид

$$
\begin{aligned}
\delta_{4} \frac{d \chi}{d \tau} & =\left(\Lambda \sin ^{2} \theta+\cos ^{2} \theta\right) \chi_{, z z}-(\Lambda-1) \sin 2 \theta \chi_{, z} \theta_{, z} \\
& +(\Lambda-1) \frac{d}{L}\left[\sin 2 \theta \chi_{, x z}-\cos 2 \theta\left(\chi_{, x} \theta_{, z}+\chi_{, z} \theta_{, x}\right)\right]
\end{aligned}
$$

где $\Lambda=\lambda_{\|} / \lambda_{\perp}, \chi \equiv \chi(x, z, \tau)=T(x, z, \tau) / T_{\mathrm{NI}}-$ безразмерная температура, а $\delta_{4}=\frac{\rho C_{p} K_{1}}{\gamma_{1} \lambda_{\perp}}-$ еще один параметр ЖК-системы.

Теперь процесс релаксации поля директора в ПОЖК наноразмерном канале к его равновесному распределению по сечению канала под действием электрических, вязких, упругих и термомеханических сил может быть описан системой нелинейных дифференциальных уравнений в частных производных (10)-(13), с учетом соответствующих граничных условий для угла

$$
\theta_{0 \leq x<1, z=-1}=\frac{\pi}{2}, \quad \theta_{x= \pm 1,-1<z<1}=\frac{\pi}{2},
$$

скорости

$$
\mathbf{v}_{-1<x<10, z= \pm 1}=\mathbf{v}_{x= \pm 1,-1<z<1}=0
$$

и температуры

$$
\begin{gathered}
\chi_{-1<x<1, z=1}=\chi_{w}, \\
\chi_{-1 \leq x \leq 1, z=-1}=\chi_{x=-1,-1<z<1}=\chi_{x=1,-1<z<1}=\chi_{w}, \\
\left(\frac{\partial \chi(x, z, \tau)}{\partial z}\right)_{0 \leq x \leq 1, z=1}=\bar{q} .
\end{gathered}
$$

Здесь $\chi_{w}=T_{w} / T_{\mathrm{NI}}$ - значение безразмерной температуры на ограничивающих поверхностях, $\bar{q}=-\frac{q d}{T_{\mathrm{NI}} \lambda_{\perp}}-$ безразмерный тепловой поток через верхнюю ограничивающую поверхность в ЖК-фазу. Следует отметить, что верхняя черта в обозначениях теплового потока $q$ в дальнейшем будет опущена.

Систему нелинейных уравнений (10)-(13), с граничными условиями (14)-(16), следует дополнить начальным условием для полярного угла

$$
\theta(\tau=0, x, z)=\theta_{\mathrm{el}}(x, z),
$$

где значение полярного угла $\theta_{\text {elast }}(x, z)-$ есть решение уравнения (10), полученное только с учетом упругих сил (при отсутствии как поля скорости, так и температуры $\left.u=w=\chi_{, x}=\chi_{, z}=0\right)$ и граничного условия (14).

\section{3. Решение гидродинамических уравнений и основные результаты}

Для того чтобы понять, какую роль играют как тепловой поток $\mathbf{q}=q \hat{\mathbf{k}}$, направленный в ЖК-канал через ограничивающую поверхность, так и двойной электрический слой с плотностью заряда $\sigma$ в формировании гидродинамического течения в наноразмерном ПОЖК-канале, предположим, что лазерное излучение сфокусировано на верхней ограничивающей поверхности. Здесь функция $q$ имеет вид

$$
q(x, z)=q_{0} \exp \left(-2 \frac{\left(x-x_{0}\right)^{2}+\left(z-z_{0}\right)^{2}}{\Delta}\right) \mathscr{H}\left(\tau_{\text {in }}-\tau\right)
$$

и описывает распределение инжектируемой энергии в наноразмерный ЖК-канал, в то время как $q_{0}$ - безразмерная мощность теплового потока, $\Delta=\frac{\omega_{0}}{d}-$ размер Гауссового пятна лазерного излучения, $x_{0}$ и $z_{0}-$ координаты его центра соответственно, $\mathscr{H}\left(\tau_{\text {in }}-\tau\right)-$ функция Хэвисайда и $\tau_{\text {in }}$ - характерное безразмерное время накачки лазерного излучения в ЖК-фазу. Следует отметить, что первые три параметра $\delta_{1}=\left(\frac{E_{\text {th }}}{\pi E_{0}}\right)^{2}$, $\delta_{2}=\xi \frac{T_{N I}}{K_{1}} \delta_{1}$ и $\delta_{3}=\rho \frac{K_{1}}{\gamma_{1}^{2}} \delta_{1}^{2}$, входящие в систему нелинейных уравнений $(10)-(13)$, зависят от величины порогового электрического поля $E_{\mathrm{th}}=\frac{\pi}{d} \sqrt{\frac{K_{1}}{\epsilon_{0} \epsilon_{a}}}$, которое, в свою очередь, обратно пропорционально ширине $d$ наноразмерного ЖК-канала. Поскольку в наших исследованиях безразмерная ширина ЖК-канала будет изменяться как $D=d / \lambda_{D}=2.0,5.0$, и 10.0 , 

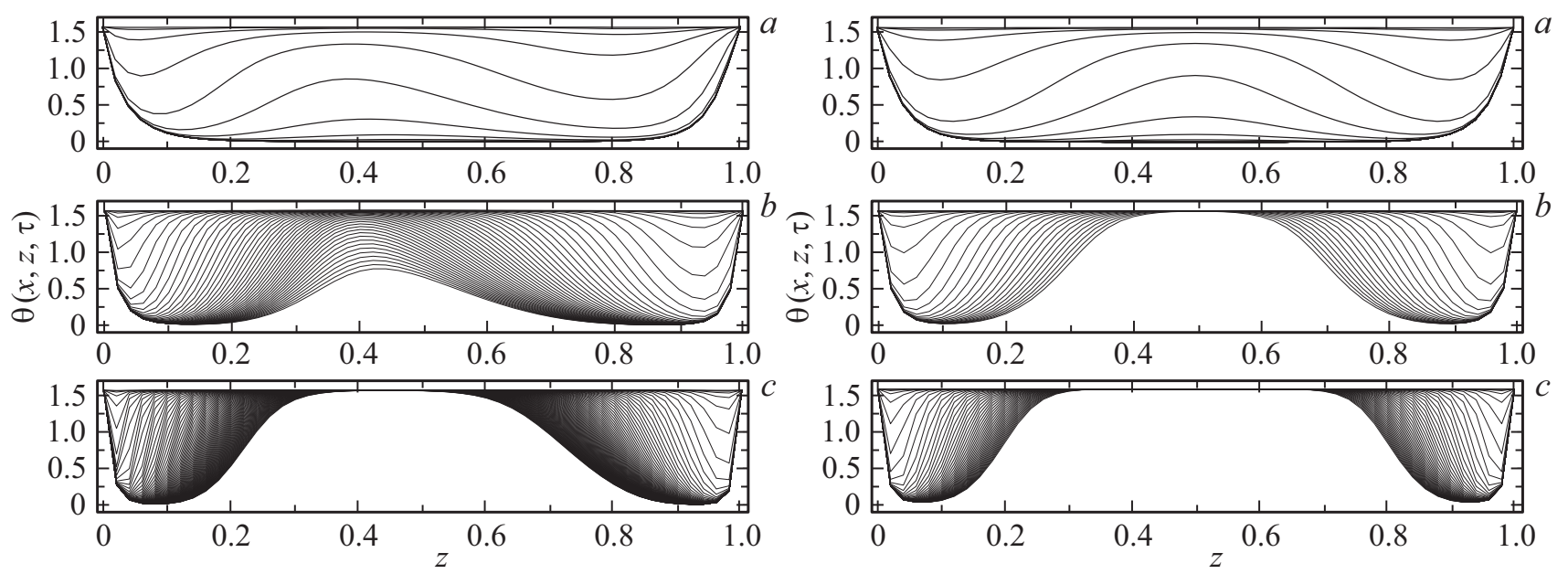

Рис. 1. Слева - эволюция распределения угла $\theta(x=0.5, z, \tau)$ по сечению наноразмерного ПОЖК-канала под действием как лазерного излучения, так и статического электрического поля $E_{0}$, для трех значений безразмерной ширины ЖК-канала: $(a) D=2.0$, (b) 5.0, и (c) 10.0 соответственно. Справа — то же что слева, только под действием электрического поля $E_{0}$.

то в случае ЖК-материала, образованного молекулами 4-cyano-4'-pentylbiphenyl (5CB) и при температуpax, соответствующих интервалу существования нематической фазы этого ЖК-соединения, значения величин порогового электрического поля изменяются как $E_{\text {th }}(D=2.0)=1.15 \cdot 10^{-3}, E_{\text {th }}(D=5.0)=0.47 \cdot 10^{-3}$, и $E_{\mathrm{th}}(D=10.0)=0.235 \cdot 10^{-3}$ (рассчитанных в единицах $\mathrm{C} / \mathrm{m}^{2}$ ) соответственно безразмерным значениям ширины, в то время как значения параметра $\delta_{1}$ изменяются соответственно как $\delta_{1}(D=2.0) \sim 9.0 \cdot 10^{-4}$, $\delta_{1}(D=5.0) \sim 1.4 \cdot 10^{-4}$, и $\delta_{1}(D=10.0) \sim 0.36 \cdot 10^{-4}$. В свою очередь, значения параметров $\delta_{2}$ и $\delta_{3}$ соответственно изменяются как $\delta_{2}(D=2.0) \sim 0.27$ и $\delta_{3}(D=2.0) \sim 1.8 \times 10^{-12}, \quad \delta_{2}(D=5.0) \sim 4.2 \cdot 10^{-2} \quad$ и $\delta_{3}(D=5.0) \sim 4.4 \cdot 10^{-14}$, и $\delta_{2}(D=10.0) \sim 1.1 \cdot 10^{-3}$ и $\delta_{3}(D=10.0) \sim 2.9 \cdot 10^{-15}$. Таким образом, величина параметра $\delta_{1}(D=2.0)$ в 25 раз больше величины $\delta_{1}(D=10.0)$, в то время как величина параметра $\delta_{3}(D=2.0)$ приблизительно в 245 раз больше чем величина $\delta_{3}(D=10.0)$. Впоследствии мы покажем, как вариации величин параметров $\delta_{i}(i=1, \ldots, 4)$, входящих в систему нелинейных уравнений в частных производных (10)-(13), будут влиять на характер гидродинамического течения, возникающего в наноразмерном ПОЖК-канале, как под действием теплового потока $\mathbf{q}=q \hat{\mathbf{k}}$, направленного в ЖК-канал через ограничивающую поверхность, так и двойного электрического слоя с плотностью заряда $\sigma$.

Процесс эволюции поля директора $\hat{\mathbf{n}}$ к его распределению $\hat{\mathbf{n}}_{\text {in }}\left(x, z, \tau_{\text {in }}\right)$, соответствующему окончанию процесса прогревания ЖК-фазы, описывающейся полярным углом $\theta(x, z, \tau)$, с его начального распределения $\theta(x, z, \tau=0)$ и до $\theta_{\text {in }}\left(x, z, \tau_{\text {in }}\right)$, а также формирование гидродинамического течения в наноразмерном ПОЖК-канале под действием как лазерного излучения, сфокусированного на верхней границе, так и двойного электрического слоя с плотностью заряда $\sigma$, были исследованы численно методом релаксаций [11], а критерием сходимости итерационной процедуры был выбран $\epsilon=\left|\left(\theta_{\mathrm{m}+1}-\theta_{\mathrm{m}}\right) / \theta_{\mathrm{m}}\right| \sim 10^{-4}$. Здесь $m-$ номер итерации.

На рис. 1 представлены результаты расчета эволюции полярного угла $\theta(x=0.5, z, \tau)$, начиная с его начального распределения $\theta(x=0.5, z, \tau=0)=$ $=\theta_{\text {elast }}(x=0.5, z) \quad$ и до $\theta_{\text {in }}\left(x, z, \tau_{\text {in }}\right)$, соответствующего окончанию процесса нагревания лазерным излучением, для трех значений безразмерной ширины ЖК-канала: (a) $D=d / \lambda_{D}=2.0 \quad(d \sim 100 \mathrm{~nm})$, (b) $D=5.0(d \sim 0.25 \mu \mathrm{m})$, и $(c) D=10.0(d \sim 0.5 \mu \mathrm{m})$ соответственно. На левой стороне рис. 1 (случай I) представлена эволюция полярного угла $\theta(x=0.5, z, \tau)$ под действием как лазерного излучения, так и двойного электрического слоя, в то время как на правой стороне рис. 1 (случай II) представлена эволюция $\theta(x=0.5, z, \tau)$ под действием только двойного электрического слоя. При этих вычислениях безразмерный тепловой поток был выбран в форме, представленной функцией $q(x, z)$ (см. (18)), с безразмерной мощностью теплового потока, равной $q_{0}=0.25\left(\sim 4.0 \cdot 10^{-2} \mathrm{~mW} / \mu \mathrm{m}^{2}\right)$ и временем накачки излучения, равным $\tau_{i n}=0.01(\sim 0.6 \mathrm{~ns})$, в то время как плотность двойного электрического слоя была выбрана равной $\sigma=10^{-2} \mathrm{C} / \mathrm{m}^{2}$. Анализируя результаты вычислений, следует отметить, что в случае I как тепловой поток мощностью $q_{0}=0.25$, так и статическое электрическое поле, создаваемое двойным электрическим слоем с плотностью заряда $\sigma=10^{-2} \mathrm{C} / \mathrm{m}^{2}$, способно деформировать поле директора таким образом, что распределение полярного угла $\theta(x=0.5, z, \tau)$ по сечению ПОЖК-канала представляет собой асимметричную функцию относительно середины сечения ЖК-канала, в то время как в случае II, под действием только электрических сил, мы имеем дело 
с симметричным распределением угла $\theta(x=0.5, z, \tau)$. Такой процесс переориентации поля директора в наноразмерном ПОЖК-канале, как он описан в случаях I и II, свидетельствует о том, что основной вклад в этот процесс переориентации осуществляется за счет статического электрического поля $E_{0}=\sigma /\left(\epsilon_{0} \epsilon_{a}\right)$, инициируемого двойным электрическим слоем. Второе, что необходимо отметить, это то, что в случае сверхтонкого ЖК-канала $D=d / \lambda_{D}=2.0(d \sim 100 \mathrm{~nm})$, плотности заряда $\sigma=10^{-2} \mathrm{C} / \mathrm{m}^{2}$ двойного электрического слоя достаточно для того, чтобы переориентировать поле директора по всей ширине ЖК-канала (см. рис. 1,a),

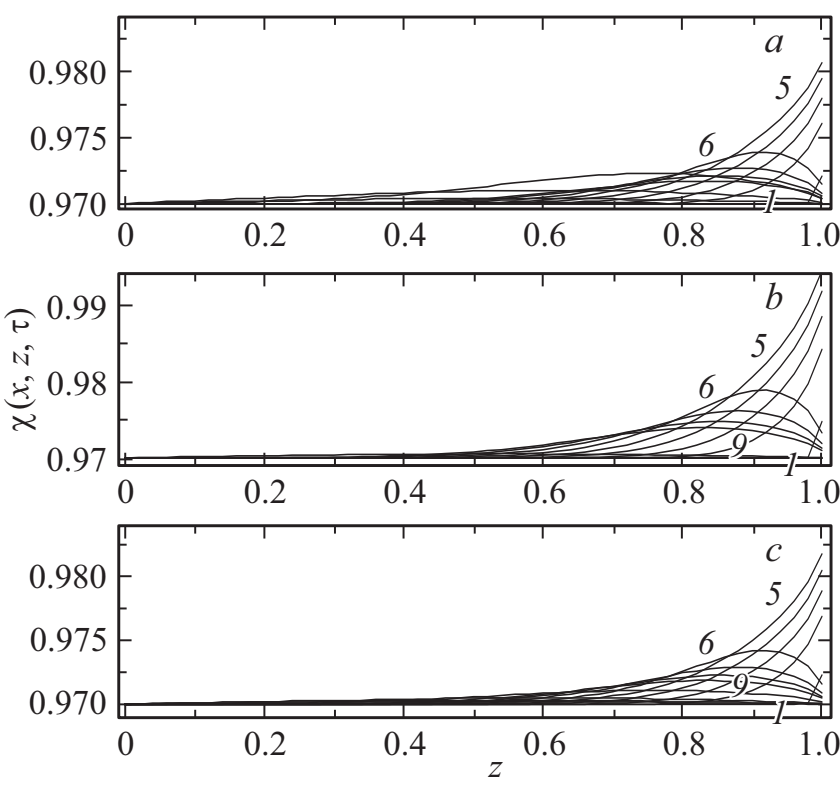

Рис. 2. Эволюция распределения поля температуры $\chi(x, z, \tau)$ по сечению сверхтонкого $(D=2.0)$ ПОЖК-канала, в процессе прогревания (кривые с 1 по 5) и охлаждения (кривые с 6 по 9) соответственно.

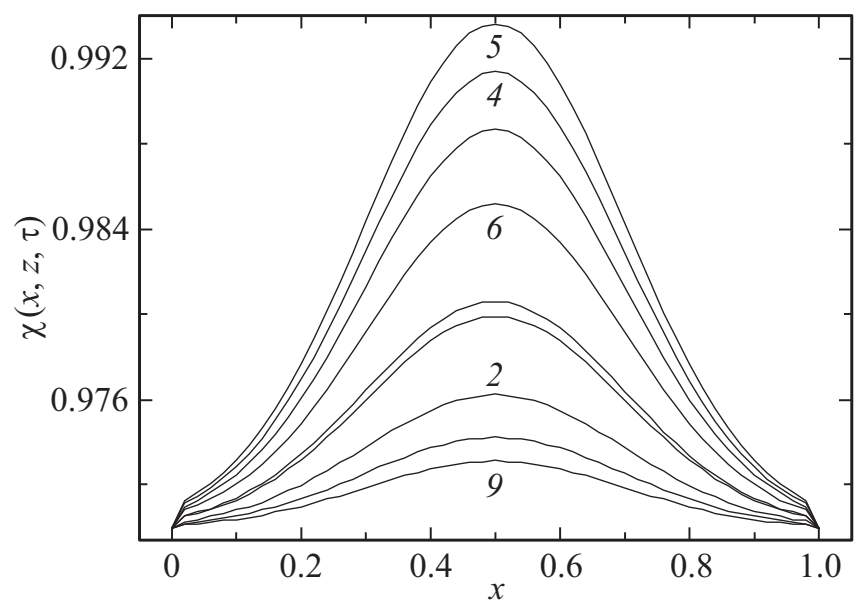

Рис. 3. Эволюция распределения поля температуры $\chi(x, z=1, \tau)$ вдоль верхней границы $(z=1)$ сверхтонкого $(D=2.0)$ ПОЖК-канала. Номера кривых соответствуют тем процессам, что показаны на рис. 2.

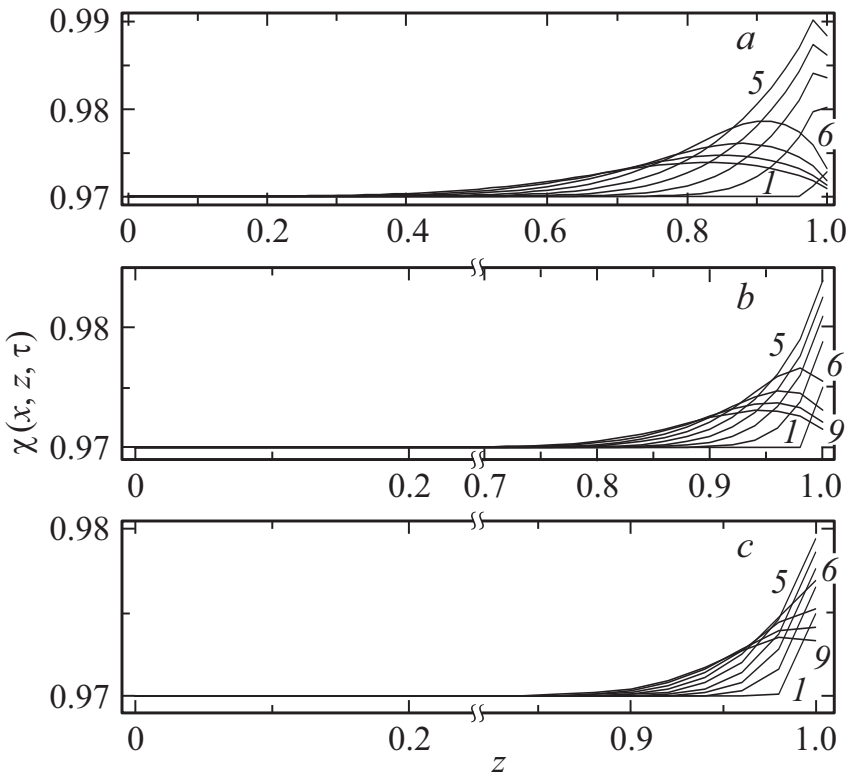

Рис. 4. Эволюция распределения поля температуры $\chi(x=0.5, z, \tau)$ по сечению ПОЖК-канала, для трех значений безразмерной ширины канала: $(a) D=2.0,(b) 5.0$, и $(c) 10.0$ соответственно. Номера кривых соответствуют тем процессам, что показаны на рис. 2.

в то время как в случае более широкого ЖК-канала $D=10.0(d \sim 0.5 \mu \mathrm{m})$ существует полость, в центре ЖК-канала $(0.35 \leq z \leq 0.55)$, где влияние как электрического поля, так и лазерного излучения полностью отсутствует. Ниже будет показано, что более сложная картина эволюции поля директора в канале шириной $D=10.0(d \sim 0.5 \mu \mathrm{m})$, чем в сверхтонком ЖК-канале $(D=2.0(d \sim 100 \mathrm{~nm}))$, приводит к сложной пространственной зависимости термомеханической силы, ответственной за формирование гидродинамического потока в этих ЖК-каналах.

Эволюция поля температуры $\chi(x, z, \tau)$ от его начального распределения $\chi(x, z, \tau=0)=\chi_{w}$ и до значений соответствующих окончанию процесса прогревания ЖК-канала $\chi\left(x, z, \tau=\tau_{i n}\right)$, представлена на рис. 2,3 и 4. Кривые с 1 по 5 номер соответствуют временам $\tau_{k}=\Delta \tau \times(k-1) \quad(k=1, \ldots, 5 ; \quad \Delta \tau=0.0025)$ (процесс нагревания ЖК-канала), в то время как кривые с 6 по 9 номер соответствуют временам $\tau_{k}=\Delta \tau \times(k-1)(k=6, \ldots, 9 ; \Delta \tau=0.0025)$ (процесс охлаждения ЖК-канала) соответственно. Зависимости $\chi\left(x_{k}, z, \tau\right)\left(x_{k}=0.25 k(k=1,2,3)\right)$ представленные на рис. $2 a-c$ описывают эволюцию безразмерного поля температуры по сечению сверхтонкого ЖК-канала $(D=2.0(d \sim 100 \mathrm{~nm}))$, для трех значений положения пространственной переменной $x_{k}=0.25 k \quad(k=1,2,3)$. Результаты вычислений показывают, что глубина прогревания ПОЖК-канала составляет $\sim 40 \mathrm{~nm}$ в глубину ЖК-фазы со стороны верхней прогреваемой границы канала. На рис. 3 представлена эволюция распределения 
безразмерного поля температуры $\chi(x, z=1, \tau)$ вдоль верхней границы ЖК-канала как в процессе прогревания (кривые с 1 по 5 номер), так и в процессе охлаждения (кривые с 6 по 9 номер) соответственно. И наконец, на рис. 4 представлены зависимости $\chi(x=0.5, z, \tau)$ по сечению ПОЖК-канала для случая трех значений ширины канала: $(a) D=2.0(d \sim 100 \mathrm{~nm}),(b) D=5.0$
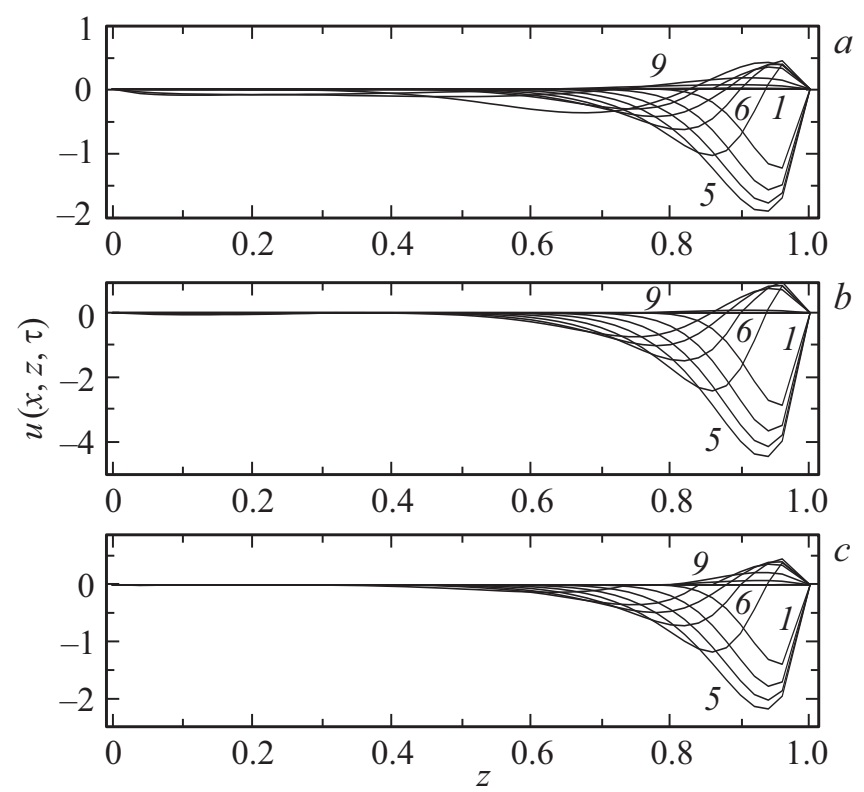

Рис. 5. Эволюция распределения горизонтальной $u(x, z, \tau)$ составляющей поля скорости $\mathbf{v}=u \hat{\mathbf{i}}+w \hat{\mathbf{k}}$ по сечению сверхтонкого $(D=2.0)$ ПОЖК-канала, для трех значений положения пространственной переменной $x_{k}=0.25 k(k=1,2,3)$. Номера кривых соответствуют тем процессам, что показаны на рис. 2.
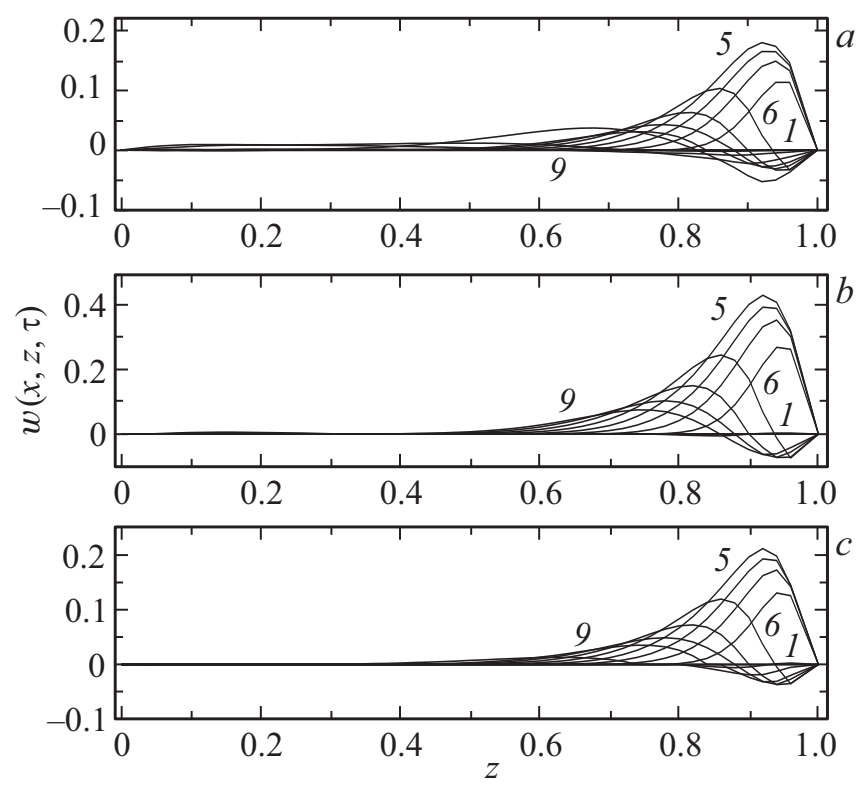

Рис. 6. Тоже что на рис. 5 , но для вертикальной $w(x, z, \tau)$ составляющей поля скорости $\mathbf{v}$. $(d \sim 0.25 \mu \mathrm{m})$, и $(c) \quad D=10.0(d \sim 0.5 \mu \mathrm{m})$ соответственно. Следует отметить, что с увеличением ширины ЖК-канала процент прогретого объема убывает с 50\%, в случае $D=2.0$, и до $10 \%$, в случае $D=10.0$ соответственно.

Эволюция распределения поля скорости $\mathbf{v}(x, z, \tau)=u(x, z, \tau) \hat{\mathbf{i}}+w(x, z, \tau) \hat{\mathbf{k}} \quad$ в $\quad$ наноразмерном ПОЖК-канале под действием как потока тепла q направленного через верхнюю границу канала, так и двойного электрического слоя представлена на рис. 5-8. На рис. 5 и 6 представлены эволюции распределения горизонтальной $u(x, z, \tau)$ (см. рис. 5) и вертикальной $w(x, z, \tau)$ (см. рис. 6) составляющих поля скорости $\mathbf{v}$ по сечению сверхтонкого ЖК-канала $D=2.0(d \sim 100 \mathrm{~nm}))$, для трех значений положения пространственной переменной $x_{k}=0.25 k(k=1,2,3)$. Кривые с 1 по 5 номер соответствуют временам $\tau_{k}=\Delta \tau \times(k-1)(k=1, \ldots, 5 ; \Delta \tau=0.0025)$ (процесс нагревания ЖК-канала), в то время как кривые с 6 по 9 номер соответствуют временам $\tau_{k}=\Delta \tau \times(k-1)$ $(k=6, \ldots, 9 ; \quad \Delta \tau=0.0025) \quad$ (процесс охлаждения ЖК-канала) соответственно.

Прежде всего следует отметить, что эволюция горизонтальной составляющей поля скорости $u$ в сверхтонком ЖК-канале $(D=2.0)$ свидетельствует о том, что вблизи верхней более теплой границы канала формируется отрицательно направленный горизонтальный поток ЖК-материала. При этом максимальное значение $|u| \sim 4.0(\sim 7.0 \mathrm{~nm} / \mathrm{ns})$ достигается вблизи точки $x=0.5, z=0.95$, что на порядок больше величины вертикальной составляющей поля скорости $|w| \sim 0.4 \quad(\sim 0.7 \mathrm{~nm} / \mathrm{ns})$. При этом, в случае сверхтонкого ЖК-канала, практически $60 \%(0 \leq z \leq 0.60)$ объема ЖК-фазы, отсчитанного от нижней границы канала, остается практически неподвижным. Следует также отметить, что в процессе охлаждения ЖК-канала скорость $\mathbf{v}=u \hat{\mathbf{i}}+w \hat{\mathbf{k}}$ стремится к нулю, а поле температуры выравнивается по всему ЖК-каналу, спустя время $\tau_{R}=0.1(\sim 6.0 \mathrm{~ns})$.

C ростом ширины ЖК-канала, с величины $D=2.0 \quad(d \sim 100 \mathrm{~nm}) \quad$ до $\quad D=10.0 \quad(d \sim 0.5 \mu \mathrm{m})$, картина эволюции поля скорости $\mathbf{v}$ под влиянием градиентов поля директора $\nabla \hat{\mathbf{n}}$ и температуры $\nabla \chi \quad$ претерпевает существенное изменение (см. рис. 7 и 8). Прежде всего абсолютная величина как горизонтальной $u$, так и вертикальной $w$ составляющих вектора скорости $\mathbf{v}$ возрастает приблизительно в 5 раз. Во вторых, гидродинамический поток инициируемый термомеханической силой формирует вихревое течение направленное по часовой стрелке, с центром вращения в точке $x=0.5, z=0.5$. В случае $D=10.0$ найбольшее абсолютное значение горизонтальной составляющей вектора скорости $|u| \sim 20.0(\sim 0.5 \mu \mathrm{m} / \mathrm{ns})$ (см. рис. 7), в то время как найбольшее абсолютное значение вертикальной составляющей вектора скорости $|w| \sim 2.0$ $(\sim 0.05 \mu \mathrm{m} / \mathrm{ns})$ (см. рис. 8) соответственно. За такое поведение поля скорости $\mathbf{v}=u \hat{\mathbf{i}}+w \hat{\mathbf{k}} \quad$ с $\quad$ ростом 

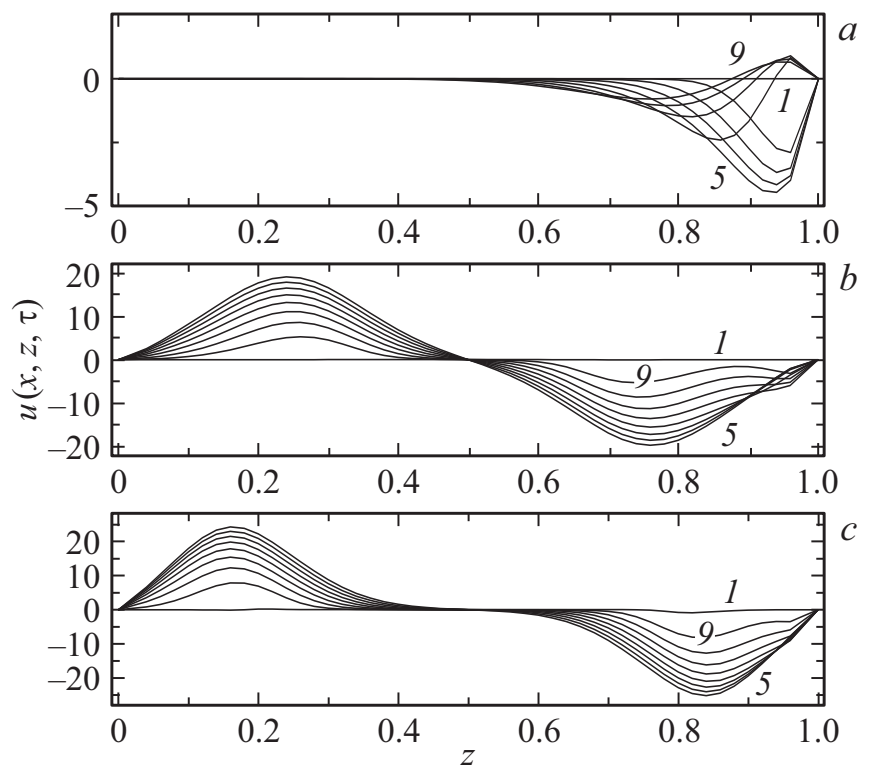

Рис. 7. Эволюция распределения горизонтальной $u(x, z, \tau)$ составляющей поля скорости $\mathbf{v}$ по сечению ПОЖК-канала, для трех значений безразмерной ширины канала: $(a) D=2.0$, (b) 5.0 и (c) 10.0 соответственно. Номера кривых соответствуют тем процессам, что показаны на рис. 2.
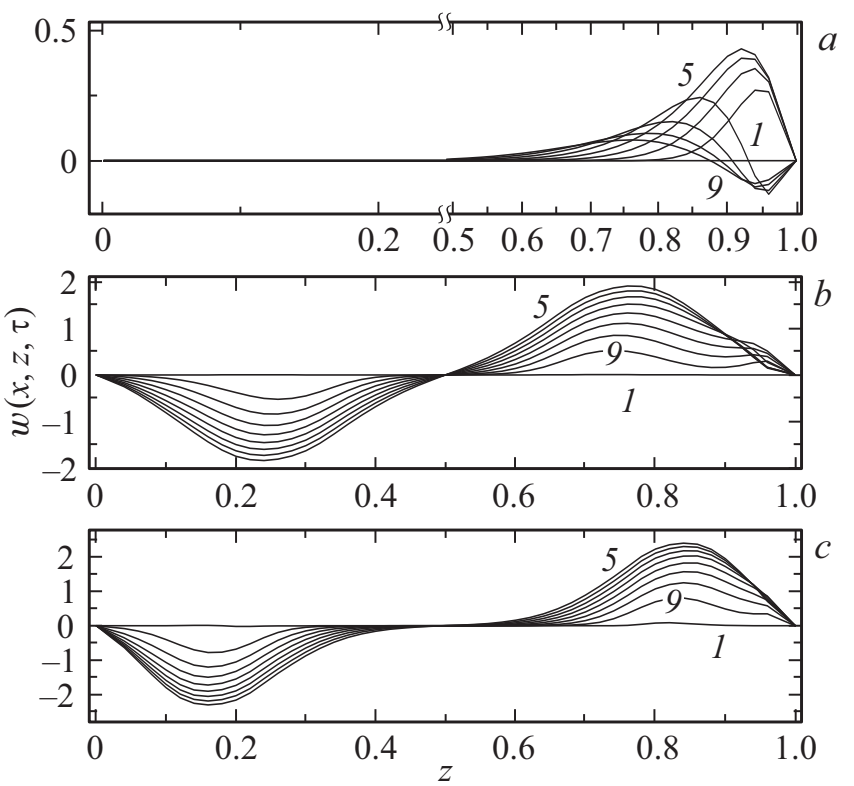

Рис. 8. Тоже что на рис. 7, но для вертикальной $w(x, z, \tau)$ составляющей поля скорости $\mathbf{v}$.

ширины ЖК-канала ответственна термомеханическая сила, обусловленная взаимодействием градиентов поля директора $\nabla \hat{\mathbf{n}}$ и температуры $\nabla \chi$. Следует отметить, что эволюция полярного угла $\theta(x=0.5, z, \tau)$ (см. рис. 1) в случае сверхтонкого ЖК-канала характеризуется выпуклым профилем $\theta(x, z, \tau)$ по сечению канала на временах $\tau_{i n}=0.01(\sim 0.6 \mathrm{~ns})$, в то время как в случае более широкого ЖК-канала
$(D=10.0 \quad(d \sim 0.5 \mu \mathrm{m}))$ эволюция полярного угла $\theta(x=0.5, z, \tau)$ характеризуется двумя практически симметричными „Горбами“ (см. рис. $1(b)$ и $(c)$ ). В результате более сложной эволюции поля директора, c ростом ширины ЖК-канала, формируется более сложная картина эволюции градиента поля директора $\nabla \hat{\mathbf{n}}$. Результаты вычислений показывают, что основным фактором, влияющим на эволюцию градиента поля директора, является статическое электрическое поле, формирующееся на границах ЖК-канала. Так, в случае более широкого ЖК-канала $(D=10.0)$, под действием двойного электрического слоя с плотностью заряда $\sigma=10^{-2} \mathrm{C} / \mathrm{m}^{2}$, внутри канала формируется полость $(0.35 \leq z \leq 0.55)$, где влияние как электрических сил, так и лазерного излучения пренебрежимо мало. А поскольку прогревание ЖК-канала посредством лазерного излучения простирается вглубь только на $40 \mathrm{~nm}$, то величины разнонаправленных горизонтальных потоков, которые формируются вблизи верхней и нижней ограничивающих поверхностей, достаточно для формирования вихревого потока в более широком ЖК-канале.

Как уже выше было отмечено, величина параметра $\delta_{1}(D=2.0)$ в 25 раз больше величины $\delta_{1}(D=10.0)$, в то время как величина параметра $\delta_{3}(D=2.0)$ приблизительно в 245 раз больше, чем величина $\delta_{3}(D=10.0)$. На величину этих параметров влияет величина порогового напряжения $E_{\mathrm{th}}=\frac{\pi}{d} \sqrt{\frac{K_{1}}{\epsilon_{0} \epsilon_{a}}}$, которая, в свою очередь, обратно пропорциональна ширине $d$ наноразмерного ЖК-канала. Поскольку деформация изначально однородно ориентированного ЖК-канала, при наложении внешнего, перпендикулярно направленного электрического поля $\mathbf{E}$, возникает только при полях $E>E_{\mathrm{th}}$, то ширина $d$ играет главную роль в процессе формирования градиента поля директора $\nabla \hat{\mathbf{n}}$ в тонких и сверхтонких ЖК-каналах. Таким образом, величина $E_{\mathrm{th}}$, а вслед за ней и ширина ЖК наноканала ответственны за столь широкий разброс значений параметров $\delta_{i}(i=1,2,3)$, входящих в систему нелинейных дифференциальных уравнений в частных производных, описывающих как процесс переориентации поля директора, так и формирования поля скорости и поля температуры.

\section{4. Заключение}

В предлагаемой работе численными методами, в рамках нелинейного обобщения классической теории Эриксена-Лесли, с учетом баланса энтропии, описано несколько сценариев формирования гидродинамических течений в наноразмерном планарно ориентированном жидкокристаллическом (ПОЖК) канале под действием как сфокусированного лазерного излучения, так и с учетом двойного электрического слоя, естественно возникающего на границе раздела ЖК-фаза/твердое тело. Учет термомеханических вкладов в выражении для сдви- 
гового напряжения и в уравнении баланса энтропии позволил описать процесс формирования вихревого потока в тонком ПОЖК-канале.

Исследованные в работе особенности, связанные с реакцией ЖК-материала, инкапсулированного в тонкие и сверхтонкие каналы, на локально формирующиеся градиенты температуры и поля директора, необходимо учитывать при создании сенсоров и датчиков используемых в биотехнологических приложениях, медицине и биометрических оптических системах.

\section{Приложение}

Уравнение баланса моментов, действующих на единицу объема ЖК-фазы, имеет вид

$$
\mathbf{T}_{\mathrm{el}}+\mathbf{T}_{\text {elast }}+\mathbf{T}_{\mathrm{vis}}+\mathbf{T}_{\mathrm{tm}}=0,
$$

где $\mathbf{T}_{\mathrm{el}}=-\epsilon_{0} \epsilon_{a} \mathbf{E} \times \hat{\mathbf{n}}(\mathbf{E} \cdot \hat{\mathbf{n}})-$ электрический, $\mathbf{T}_{\text {elast }}=$ $=\frac{\delta \mathscr{W}_{\text {elast }}}{\delta \hat{\mathbf{n}}} \times \hat{\mathbf{n}}-$ упругий, $\mathbf{T}_{\text {vis }}=\frac{\delta \mathscr{R}^{\text {vis }}}{\delta \hat{\mathbf{n}}_{t}} \times \hat{\mathbf{n}}-$ вязкий и $\mathbf{T}_{\mathrm{tm}}=\frac{\delta \mathscr{R}^{\mathrm{tm}}}{\delta \hat{\mathbf{n}}_{\mathrm{t}}} \times \hat{\mathbf{n}}-$ термомеханический вклады в баланс моментов соответственно. Здесь $\hat{\mathbf{n}}_{, \mathrm{t}} \equiv \frac{d \hat{\mathbf{n}}}{d t}-$ материальная производная вектора $\hat{\mathbf{n}}=n_{x} \hat{\mathbf{i}}+n_{z} \hat{\mathbf{k}}$.

Уравнение баланса линейных моментов, действующих на единицу объема ЖК-фазы, имеет вид

$$
\rho \frac{d \mathbf{v}}{d t}=\nabla \cdot \sigma
$$

где $\frac{d \mathbf{v}}{d t}=\frac{\partial v}{\partial t}+u v_{, \mathrm{x}}+w v_{\text {, }}, \sigma=\sigma^{\mathrm{el}}+\sigma^{\mathrm{vis}}+\sigma^{\mathrm{tm}}-\mathscr{P} \mathscr{E}-$ полное выражение для тензора напряжения (ТН), состоящее из упругого $\sigma^{\mathrm{el}}=-\frac{\partial \mathscr{W}_{\mathrm{el}}}{\partial \nabla \hat{\mathbf{n}}} \cdot(\nabla \hat{\mathbf{n}})^{\mathrm{T}}, \quad$ вязкого $\sigma^{\text {vis }}=\frac{\delta \mathscr{R}^{\text {vis }}}{\delta \nabla \mathbf{v}}$ и термомеханического $\sigma^{\mathrm{tm}}=\frac{\delta \mathscr{R}^{\mathrm{tm}}}{\delta \nabla \mathbf{v}}$ вкладов в ТН соответственно. Здесь $\mathscr{R}=\mathscr{R}^{\text {vis }}+\mathscr{R}^{\text {tm }}+\mathscr{R}^{\text {th }}-$ полная диссипационная функция Рэлея, $\mathscr{W}_{\mathrm{el}}=\frac{1}{2}\left[K_{1}(\nabla \cdot \hat{\mathbf{n}})^{2}\right.$ $\left.+K_{3}(\hat{\mathbf{n}} \times \nabla \times \hat{\mathbf{n}})^{2}\right]$ - плотность упругой энергии, $K_{1}$ и $K_{3}$ - продольный и поперечный коэффициенты упругости, $\mathscr{P}$ - гидростатическое давление в ЖК-системе, $\mathscr{E}$ - единичный тензор, в то время как $\mathscr{R}^{\text {vis }}=$ $=\alpha_{1}\left(\hat{\mathbf{n}} \cdot \mathbf{D}_{\mathrm{s}} \cdot \hat{\mathbf{n}}\right)^{2}+\gamma_{1}\left(\hat{\mathbf{n}}_{\mathrm{t}}-\mathbf{D}_{\mathrm{a}} \cdot \hat{\mathbf{n}}\right)^{2}+2 \gamma_{2}\left(\hat{\mathbf{n}}_{\mathrm{t}}-\mathbf{D}_{\mathrm{a}} \cdot \hat{\mathbf{n}}\right)\left(\mathbf{D}_{\mathrm{s}} \cdot \hat{\mathbf{n}}\right.$ $\left.-\left(\hat{\mathbf{n}} \cdot \mathbf{D}_{\mathrm{s}} \cdot \hat{\mathbf{n}}\right) \hat{\mathbf{n}}\right)+\alpha_{4} \mathbf{D}_{\mathrm{s}}: \mathbf{D}_{\mathrm{s}}+\left(\alpha_{5}+\alpha_{6}\right)\left(\hat{\mathbf{n}} \cdot \mathbf{D}_{\mathrm{s}} \cdot \mathbf{D}_{\mathrm{s}} \cdot \hat{\mathbf{n}}\right)-$ вязкий, $\quad$ a $\quad \frac{1}{\xi} \mathscr{R}^{\mathrm{tm}}=(\hat{\mathbf{n}} \cdot \nabla T) \mathbf{D}_{\mathrm{s}}: \mathbf{M}+\nabla T \cdot \mathbf{D}_{\mathrm{s}} \cdot \mathbf{M} \cdot \hat{\mathbf{n}}$ $+(\hat{\mathbf{n}} \cdot \nabla T)\left(\hat{\mathbf{n}}_{\mathrm{t}}-\mathbf{D}_{\mathrm{a}} \cdot \hat{\mathbf{n}}-3 \mathbf{D}_{\mathrm{s}} \cdot \hat{\mathbf{n}}+3\left(\hat{\mathbf{n}} \cdot \mathbf{D}_{\mathrm{s}} \cdot \hat{\mathbf{n}}\right) \hat{\mathbf{n}}\right) \cdot \mathbf{M} \cdot \hat{\mathbf{n}}$ $+\hat{\mathbf{n}}(\nabla \mathbf{v})^{\mathrm{T}} \cdot \mathbf{M} \cdot \nabla T+\frac{1}{2}\left(\hat{\mathbf{n}} \cdot \mathbf{D}_{\mathrm{s}} \cdot \hat{\mathbf{n}}\right) \nabla T \cdot \mathbf{M} \cdot \hat{\mathbf{n}}+\hat{\mathbf{n}}_{\mathrm{t}} \cdot \mathbf{M} \cdot \nabla T$ $+\frac{1}{2} \mathscr{M}_{0} \nabla T \cdot \nabla \mathbf{v} \cdot \hat{\mathbf{n}}+(\hat{\mathbf{n}} \cdot \nabla T) \mathscr{M}_{0}\left(\hat{\mathbf{n}} \cdot \mathbf{D}_{\mathrm{s}} \cdot \hat{\mathbf{n}}\right)$ $+\frac{1}{2} \mathscr{M}_{0} \hat{\mathbf{n}}_{\mathrm{t}} \cdot \nabla T-$ термомеханический $\quad$ и $\quad \mathscr{R}^{\mathrm{th}}=\frac{1}{T}$ $\times\left(\lambda_{\|}(\hat{\mathbf{n}} \cdot \nabla T)\right)^{2}+\lambda_{\perp}\left(\nabla T-\hat{\mathbf{n}}(\hat{\mathbf{n}} \cdot \nabla T)^{2}\right)-$ термический вклады в полное выражение для функции Рэлея $\mathscr{R}$ соответственно. Здесь $\alpha_{1}-\alpha_{6}-$ коэффициенты вязкости Лесли, $\gamma_{1}(T)$ и $\gamma_{2}(T)$ - коэффициенты вращательной вязкости ЖК-системы, $\xi$ - термомеханическая постоянная, $\lambda_{\|}$и $\lambda_{\perp}$ - коэффициенты теплопроводности
ЖК-системы вдоль и поперек направления директора $\hat{\mathbf{n}}$ соответственно. Тензоры $\mathbf{D}_{\mathrm{s}}=\frac{1}{2}\left[\nabla \mathbf{v}+(\nabla \mathbf{v})^{\mathrm{T}}\right] \quad$ и $\mathbf{D}_{\mathrm{a}}=\frac{1}{2}\left[\nabla \mathbf{v}-(\nabla \mathbf{v})^{\mathrm{T}}\right]-$ являются симметричным и асимметричным вкладами в тензор скорости деформации, $\quad \mathbf{M}=\frac{1}{2}\left[\nabla \hat{\mathbf{n}}+(\nabla \hat{\mathbf{n}})^{\mathrm{T}}\right] \quad$ и $\quad \mathscr{M}_{0}=\nabla \cdot \hat{\mathbf{n}} \quad-$ скалярный инвариант тензора М.

Уравнение теплопроводности описывающее изменение поля температуры $T(x, z, t)$ под действием потока тепла q через верхнюю границу ПОЖК-канала имеет вид

$$
\rho C_{P} \frac{d T}{d t}=-\nabla \cdot \mathbf{Q}
$$

где $\mathbf{Q}=-T \frac{\delta \mathscr{R}}{\delta \nabla T}-$ есть тепловой поток в ЖК-системе, а $C_{P}$ - коэффициент теплоемкости соответственно.

\section{Финансирование работы}

Работа выполнена при финансовой поддержке Минобрнауки (гранты 3.11888.2018/11.12 и 3.9585.2017/8.9) и при финансовой поддержке РФФИ и Немецкого научноисследовательского сообщества в рамках научного проекта № 20-52-12040.

\section{Список литературы}

[1] S.J. Woltman, G.D. Jay, G.P. Crawford. Nature Mater. 6, 929 (2007).

[2] J.G. Cuennet, A.E. Vasdekis, L. De Sio, D. Plaltis. Nature Photonic. 5, 234 (2011).

[3] A.P.H.J. Schenning, G.P. Crawford, D.J. Broer. Liquid Crystal Sensors. CRC Press, Taylor and Francis Group, Boca Raton (2018). 164 p.

[4] T.M. Squires, S.R. Quake. Rev. Mod. Phys. 77, 977 (2005).

[5] P.G. de Gennes, J. Prost. The Physics of Liquid Crystals. 2nd ed. Oxford University Press, Oxford (1995).

[6] A.V. Zakharov, A.A. Vakulenko. J. Chem. Phys. 127, 084907 (2007).

[7] J.N. Israellachvili. Intermolecular and Surface Forces. Academic Press, London (1992). 450 p.

[8] J.L. Ericksen. Arch. Ration. Mech. Anal. 4, 231 (1960).

[9] F.M. Leslie. Arch. Ration. Mech. Anal. 28, 265 (1968).

[10] Л.Д. Ландау, Е.М. Лифшиц. Гидродинамика. Наука, М. (1986). $736 \mathrm{c}$.

[11] И.С. Березин, Н.Р. Жидков. Методы вычислений. Физматгиз, М. (1964). 464 с.

Редактор Ю.Э. Китаев 